\title{
INTERFERÊNCIA DE HERBICIDAS NO DESENVOLVIMENTO DO MILHETO
}

\author{
INTERFERENCE OF HERBICIDES IN THE MILLET DEVELOPMENT \\ Eduardo Paggotto ${ }^{\text {a* }}$, Fernando Machado dos Santos ${ }^{\text {a }}$, Henrique Solagna Kraemer ${ }^{\mathrm{a}}$, Eduardo Carlos Ruedell ${ }^{\mathrm{a}}$, Dieferson Frandaloso ${ }^{\mathrm{a}}$. \\ ${ }^{\text {a}}$ Setor de Culturas Anuais, Instituto Federal de Educação Ciência e Tecnologia do Rio Grande do Sul, Campus Sertão, Rio Grande do Sul, Brasil. \\ *Autor correspondente: adropaggotto@hotmail.com.
}

\section{INFORMAÇÕES DO ARTIGO}

\section{Histórico do artigo:}

Recebido: 04 Setembro 2020.

Aceito: 03 Fevereiro 2021.

Publicado: 31 Março 2021.

\section{Palavras-chave/Keywords:}

Fitotoxicidade/ Phytotoxicity.

Gramíneas/ Grasses.

Seletividade/ Selectivity.

\section{Financiamento:}

Instituto Federal de Educação, Ciência e Tecnologia do Rio Grande do Sul Campus Sertão.

Direito Autoral: Este é um artigo de acesso aberto distribuído sob os termos da Licença Creative Commons, que permite uso, distribuição e reprodução irrestritos em qualquer meio, desde que $\mathrm{o}$ autor $\mathrm{e}$ a fonte originais sejam creditados.

\section{Citação deste artigo:}

PAGGOTTO, E.; SANTOS, F. M.; KRAEMER, H. S.; RUEDELL, E. C.; FRANDALOSO, D. Interferência de herbicidas no desenvolvimento do milheto. Revista Brasileira de Herbicidas, v. 19, n. 4. 2020.

\begin{abstract}
RESUMO
O milheto é uma gramínea anual de estação quente que possui porte ereto e alto, usado como fonte de alimento na pecuária e como cobertura de solo no sistema de plantio direto. Por ser uma cultura de baixo valor econômico são raras as informações a seu respeito. O objetivo do trabalho foi verificar a seletividade dos herbicidas atrazine e 2,4-D aplicados isolados ou em associação em diferentes épocas na cultura do milheto. $\mathrm{O}$ experimento foi realizado a campo em parcelas de $12 \mathrm{~m}^{2}$ e delineamento de blocos ao acaso, sendo 10 tratamentos e quatro repetições. Os tratamentos foram: 1- testemunha sem capina; 2- testemunha capinada; 3- atrazine em pré-semeadura; 4- atrazine em pós-semeadura; 5- atrazine em V4; 6- 2,4-D em V4; 7- 2,4-D (150\% da dose) em V4; 8- atrazine em présemeadura + 2,4-D em V4; 9- atrazine em pós-semeadura + 2,4-D em V4, 10- atrazine + 2,4-D em V4. Avaliou-se o número de plantas por metro linear, a fitotoxicidade aos 7, 14, 21 e 28 dias após aplicação, a altura de plantas e a massa seca da parte aérea. Houve redução no estande de plantas com aplicação de atrazine em pré e pós-semeadura. $\mathrm{O}$ herbicida 2,4-D apresentou maiores níveis de fitotoxicidade, menor altura de plantas e menor produção de massa seca em todas as avaliações. $\mathrm{O}$ herbicida atrazine se apresenta como uma boa alternativa de controle de plantas daninhas na cultura do milheto quando aplicado em V4.
\end{abstract}

\section{ABSTRACT}

Millet is an annual hot season grass that has an upright and tall size, used as a food source in livestock and as a soil cover in the no-tillage system. As it is a culture of low economic value, information about it is rare. The objective of the work was to verify the selectivity of the herbicides atrazine and 2,4-D applied alone or in association at different times in the millet culture. The experiment was carried out in the field in $12 \mathrm{~m} 2$ plots and randomized block design, with 10 treatments and four replications. The treatments were: 1 - control without weeding; 2- weed control; 3- atrazine in pre-sowing; 4- post-sowing atrazine; 5- atrazine in V4; 6- 2,4-D in V4; 7- 2,4-D (150\% of the dose) in V4; 8- atrazine in presowing $+2,4$-D in V4; 9- atrazine in post-sowing $+2,4$-D in V4, 10-atrazine + 2,4-D in V4. The number of plants per linear meter, phytotoxicity at 7, 14, 21 and 28 days after application, plant height and shoot dry weight were evaluated. There was a reduction in the plant stand with application of atrazine in pre and post-sowing. The herbicide 2,4-D showed higher levels of phytotoxicity, lower plant height and lower dry mass production in all evaluations. The herbicide atrazine presents itself as a good alternative for weed control in the millet culture when applied in V4. 


\section{Introdução}

O milheto (Pennisetum glaucum) é uma gramínea anual de estação quente, originária do continente africano, que apresenta porte ereto e alto, com grande potencial de perfilhamento mesmo em condições de déficit hídrico (DANTAS; NEGRÃO, 2010). Seu elevado potencial de enraizamento possibilita a extração de nutrientes mesmo em níveis baixos de fertilidade, possibilitando sua reciclagem (MARCANTE; CAMACHO; PAREDES, 2011).

Sua elevada produção de matéria seca o tornou um dos principais alimentos aos bovinos em pastagens de verão, porém, nos últimos anos, a necessidade de conservação do solo, principalmente no período de outono, para cobrir o chamado "vazio outonal" consolidou o milheto como uma das principais espécies forrageiras utilizadas com a finalidade de cobertura do solo (COSTA et al., 2014).

Apesar de ser uma espécie com elevada rusticidade, o milheto apresenta crescimento inicial lento, ficando sujeito à elevada competição com plantas daninhas. A ausência de controle das plantas daninhas no decorrer do período crítico de competição, que se estende até sete semanas após a emergência da cultura, é capaz de causar perdas de produtividade de grãos de até $36 \%$ (CARSON, 1987). O controle de plantas daninhas no milheto deve ser realizado precocemente, pois a cultura só tolera a presença de plantas daninhas após atingir de 15 a $20 \mathrm{~cm}$ de altura (BERGLUND, 2007).

Como todas as gramíneas, sua palhada apresenta elevada relação $\mathrm{C} / \mathrm{N}$, aliada à elevada produção de matéria seca, possibilita a cobertura do solo por longos períodos. Sua alta capacidade de enraizamento também é aproveitada com o intuito de melhorar a estruturação do solo e permitir a melhor infiltração de água (HERRADA; LEANDRO; FERREIRA, 2017). Por ser uma cultura com pouca exploração comercial, as pesquisas do setor privado são raras, dessa forma o cultivo do milheto carece de informações acerca do uso de herbicidas durante seu desenvolvimento e sua seletividade à cultura. (PEREIRA FILHO et al., 2003).

Os herbicidas atrazine e 2,4-D são utilizados amplamente na cultura do milho e sorgo e apresentam boa seletividade e eficiência no controle de plantas daninhas, porém para o milheto as informações são escassas. O milheto apresentou tolerância ao herbicida atrazine quando aplicado em estádios mais avançados da planta e em menores doses (DAN et al., 2011). O herbicida 2,4-D apresentou seletividade aceitável em aplicações de pósemergência na cultura do milheto (PACHECO et al., 2007).

Nesse contexto, o objetivo desse trabalho foi verificar a seletividade dos herbicidas atrazine e 2,4-D aplicados em diferentes épocas, isolados ou em associações na cultura do milheto.

\section{Materias e Métodos}

O experimento foi realizado a campo, na área experimental do IFRS - Campus Sertão, em Sertão, RS, localizado nas coordenadas $28^{\circ} 02^{\prime} \mathrm{S}$ e $52^{\circ} 15^{\prime} \mathrm{O}$ e altitude de $690 \mathrm{~m}$, durante os meses de outubro de 2019 e janeiro de 2020. O clima segundo a classificação de Köppen é do tipo Mesotérmico úmido com verão quente $(\mathrm{Cfa})$ e temperatura média de $17,6^{\circ} \mathrm{C}$ e precipitação média anual de $1700 \mathrm{~mm}$.

A dessecação da área foi realizada com o herbicida glifosato na dose de $1.080 \mathrm{~g} \mathrm{ha}^{-1}$ de e.a., doze dias antes da semeadura do milheto (cultivar BRS 1503), a qual continha aveia-preta e centeio em fase reprodutiva. A matéria seca das plantas de cobertura foi analisada a partir de coleta e secagem das amostras em estufa até o peso constante, obtendo-se $6.017,3 \mathrm{~kg} \mathrm{ha}^{-1}$.

O experimento foi conduzido em blocos ao acaso com quatro repetições, onde cada unidade experimental continha uma área de $12 \mathrm{~m}^{2}$. Foram realizados dez tratamentos: 1- testemunha sem capina; 2- testemunha capinada; 3- atrazine em pré-semeadura; 4- atrazine em póssemeadura; 5- atrazine em V4; 6- 2,4-D em V4; 7- 2,4-D ( $150 \%$ da dose) em V4; 8 - atrazine em pré-semeadura $+2,4-$ D em V4; 9- atrazine em pós-semeadura + 2,4-D em V4, 10- atrazine + 2,4-D em V4. A dose de atrazine (Atrazine Nortox $500 \mathrm{SC}, 500 \mathrm{~g} \mathrm{~L}^{-1}$ de i.a., SC, Nortox) foi de 2.500 g i.a. ha ${ }^{-1}$ e a dose de 2,4-D (2,4-D Nortox, $670 \mathrm{~g} \mathrm{~L}^{-1}$ e.a., SL, Nortox) de 1.005 g. e.a. ha ${ }^{-1}$ de equivalente ácido. Todas as parcelas que receberam tratamento com herbicidas foram capinadas com a fim de inibir uma possível interferência das plantas daninhas nas avaliações.

A semeadura da área foi realizada no dia 24/10/2019 com uma semeadora Semeato ${ }^{\circledR} 15 / 17$, contendo 17 linhas espaçadas em $17 \mathrm{~cm}$, com sistema de distribuição de sementes do tipo rotor acanelado helicoidal e de fertilizante do tipo rosca-sem-fim com transbordo. A regulagem da semeadora para semente e fertilizante mineral foi realizada em galpão e obteve-se uma quantidade de $40 \mathrm{~kg} \mathrm{ha}^{-1} \mathrm{de}$ sementes, $24 \mathrm{~kg} \mathrm{ha}^{-1}$ de nitrogênio, $84 \mathrm{~kg} \mathrm{ha}^{-1}$ de fósforo e $48 \mathrm{~kg} \mathrm{ha}^{-1}$ de potássio. A análise de solo realizada na área apresentou $\mathrm{pH}$ em água de 5,60; $\mathrm{Al}, \mathrm{Ca}, \mathrm{Mg}, \mathrm{H}+\mathrm{Al}$ e CTC (efetiva) apresentaram 0,$0 ; \quad 5,74 ; 2,35 ; \quad 3,35$ e $8,77 \mathrm{cmol}_{\mathrm{c}} \mathrm{dm}^{-3}$ respectivamente, $\mathrm{P}$ e K apresentaram teores de 10,4 e $265 \mathrm{mg} \mathrm{dm}^{3}$ respectivamente, teor de argila de $54 \%$ e $\mathrm{MO}=22 \mathrm{~g} \mathrm{dm}^{3}$.

Simultânea à semeadura, foram aplicados os primeiros tratamentos que consistiam no herbicida atrazine em pré e pós semeadura do milheto. Os herbicidas foram aplicados com pulverizador costal pressurizado com $\mathrm{CO}_{2}$, com vazão de $180 \mathrm{~L} \mathrm{ha}^{-1}$, bicos de pulverização do tipo cone vazio com espaçamento de $0,5 \mathrm{~m}$ entre si e pressão de $300 \mathrm{kPa}$. A avaliação do número de plantas por metro linear foi realizada 13 dias após a semeadura (DAS), contando as plantas de um metro das três linhas centrais de cada parcela e posteriormente calculado sua média.

A aplicação de $\mathrm{N}$ em cobertura que seria realizada no estádio de desenvolvimento V4, anterior à aplicação dos tratamentos em pós-emergência da cultura foi impossibilitada pela ausência de precipitação significativa ( $\geq 10 \mathrm{~mm}$ ) por um período de 19 dias (17 a $34 \mathrm{DAS}$ ), como comprovam os dados climáticos da Estação Meteorológica da Embrapa Trigo (EMBRAPA, 2020) em Passo Fundo RS (Figura 1). 
60.00

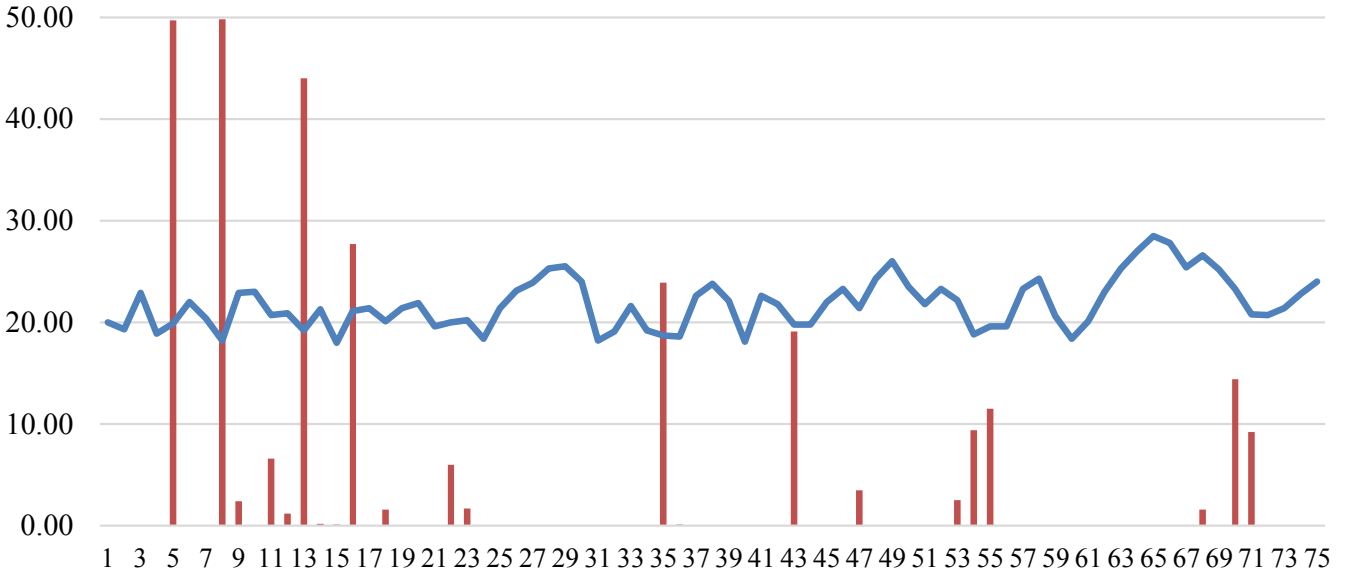

Precipitação diária $(\mathrm{mm})$

Temperatura média diária $\left({ }^{\circ} \mathrm{C}\right)$

Figura 1. Dados climáticos de precipitação e temperatura média do período de realização do experimento da Estação Meteorológica da Embrapa Trigo em Passo Fundo - RS.

A aplicação dos herbicidas no estádio de desenvolvimento V4 foi realizada 36 DAS com o mesmo pulverizador, ponta de pulverização e vazão. As avaliações de fitotoxicidade foram realizadas aos 7, 14, 21 e 28 dias após a aplicação (DAA) dos herbicidas. A avaliação foi feita utilizando-se escala percentual de 0 a 100 , em que 0 representa ausência de sintomas e 100 a morte total da planta (FRANS, 1972).

A avaliação da altura de plantas foi realizada 74 DAS, medindo 10 plantas das linhas centrais da parcela, escolhidas aleatoriamente, com o auxílio de uma fita métrica, do chão até a altura de inserção da panícula. A coleta da matéria seca das plantas foi realizada aos 74 DAS, coletando as plantas em um metro nas três linhas centrais de cada parcela. A secagem das amostras foi feita em estufa pelo método convencional, à temperatura de $65^{\circ} \mathrm{C}$ até a obtenção do peso constante das amostras (SERAFIM; ANTONELLI; SANTOS, 2016).

Todos os resultados das avaliações foram submetidos à análise estatística pelo software ASSISTAT ${ }^{\circledR} \mathrm{v} 7.7$, onde foi realizada análise de variância (ANOVA) e comparados pelo teste de Tukey a 5\% de probabilidade.

\section{Resultados e Discussões}

$\mathrm{Na}$ avaliação do número de plantas por metro linear (Tabela 1), realizada 13 dias após a semeadura, foi possível evidenciar uma redução na emergência das plântulas de milheto nos tratamentos que continham atrazine, tanto em pré quanto em pós-semeadura, 21,50 e 21,25 plantas $\mathrm{m}$ linear $^{-1}$ respectivamente, sendo que a testemunha capinada apresentou 26,00 plantas $\mathrm{m}_{\text {linear }}{ }^{-1}$. Para a cultura do sorgo, o herbicida atrazine aplicado em pré-emergência, não apresentou redução no estande de plantas e nem no desenvolvimento (RODRIGUES, 2019). O efeito da aplicação após a semeadura não apresentou diferença significativa em relação àquela realizada anteriormente à semeadura, isso porque o herbicida atrazine sofre pouca retenção pela palhada, principalmente em situações em que ocorrem precipitação em um curto período após a aplicação do herbicida, sendo facilmente carregado pela água até o solo (FORNAROLLI et al., 1998). A quantidade de palha presente na área era de $6.017,3 \mathrm{~kg} \mathrm{ha}^{-1}$, porém a precipitação de $49 \mathrm{~mm}$ ocorrida quatro dias após a aplicação da atrazine possibilitou que ela chegasse ao solo, tanto no tratamento em pré, quanto em pós semeadura.

Em relação à altura de plantas (Tabela 1), o herbicida atrazine aplicado antes da emergência do milheto, não apresentou redução significativa, porém, quando aplicado no estádio de desenvolvimento V4 causou redução no porte das plantas de $0,15 \mathrm{~m}$ em relação à testemunha, concordando com os dados encontrados em trabalho realizado por Dan et al. (2011). A aplicação do herbicida 2,4-D causou grande redução na altura das plantas de milheto, cerca de 0,39 m em relação à testemunha capinada, porém a maior dose do herbicida não apresentou diferença significativa em relação à dose normal e o mesmo associado ao herbicida atrazine em pré-emergência da cultura ou aplicado no estádio V4 não mostraram diferença em relação ao 2,4-D aplicado isoladamente. O herbicida 2,4-D aplicado principalmente em estádios iniciais nas culturas de milheto e sorgo causa redução no desenvolvimento das plantas (PACHECO et al. 2007; PETTER et al., 2011). A testemunha não capinada apresentou altura de $0,92 \mathrm{~m}$, resultado similar aos tratamentos que receberam o herbicida 2,4-D, isso se deve à alta infestação de plantas daninhas na área e ao seu lento crescimento inicial (BERGLUND, 2007). 
E. PAGGOTTO et al.

Tabela 1. Plantas por metro linear, altura de plantas e massa seca do milheto, na região Norte do Rio Grande do Sul. IFRS, Sertão, 2020.

\begin{tabular}{lccc}
\hline \multicolumn{1}{c}{ Tratamento } & Plantas.m $^{-1}$ & Altura de plantas $(\mathrm{m})$ & ${\text { Massa seca }\left(\mathrm{t} . \mathrm{ha}^{-1}\right)}^{-1}$ \\
\hline Testemunha sem capina & $27,25 \mathrm{a}^{1}$ & $0,92 \mathrm{c}$ & $3,49 \mathrm{de}$ \\
Testemunha capinada & $26,25 \mathrm{a}$ & $1,31 \mathrm{a}$ & $6,37 \mathrm{a}$ \\
Atrazine em pré-emergência & $21,50 \mathrm{bc}$ & $1,20 \mathrm{ab}$ & $6,03 \mathrm{a}$ \\
Atrazine em pós-emergência & $21,25 \mathrm{bc}$ & $1,21 \mathrm{ab}$ & $5,97 \mathrm{a}$ \\
Atrazine em V4 & $24,00 \mathrm{abc}$ & $1,16 \mathrm{~b}$ & $5,35 \mathrm{~b}$ \\
2,4-D em V4 & $25,00 \mathrm{abc}$ & $0,92 \mathrm{c}$ & $4,24 \mathrm{c}$ \\
2,4-D 150\% em V4 & $24,75 \mathrm{abc}$ & $0,82 \mathrm{c}$ & $3,43 \mathrm{e}$ \\
Atrazine em pré-emergência + 2,4-D em V4 & $21,25 \mathrm{bc}$ & $0,92 \mathrm{c}$ & $3,98 \mathrm{~cd}$ \\
Atrazine em pós-emergência + 2,4-D em V4 & $20,50 \mathrm{c}$ & $0,91 \mathrm{c}$ & $4,06 \mathrm{c}$ \\
Atrazine + 2,4-D em V4 & $25,50 \mathrm{ab}$ & $0,90 \mathrm{c}$ & $3,82 \mathrm{cde}$ \\
\hline Média & 23,72 & 1,16 & 4,67 \\
${ }^{2}$ Dms & 4,70 & 0,13 & 0,51 \\
${ }^{3} \mathrm{CV}(\%)$ & 8,14 & 5,21 & 4,52 \\
\hline
\end{tabular}

${ }^{1}$ Médias seguidas pela mesma letra minúscula na vertical não diferem significativamente pelo teste de Tukey a $5 \%$ de probabilidade. ${ }^{2}$ Dms: diferença mínima significativa. ${ }^{3} \mathrm{CV}$ : Coeficiente de Variação.

A massa seca da parte aérea (Tabela 1) dos tratamentos que receberam atrazine em pré-emergência não foi afetada em comparação à testemunha capinada, entretanto, quando aplicada em V4 apresentou redução de $16 \%$. O herbicida 2,4-D aplicado em V4 com 100\% da dose apresentou uma redução de $33 \%$ na massa seca em relação a testemunha capinada, já quando aplicado em 150\% da dose, a redução foi de $46 \%$, estes resultados corroboram com dados já encontrados, que relatam que o incremento de dose de 2,4-D propicia uma maior redução de massa seca da cultura do milheto (PACHECO et al., 2007).

Durante o período de realização do experimento o acumulado de precipitação foi abaixo da média, ocorrendo períodos prolongados sem chuva significativa, além de causar déficit hídrico à cultura, a ausência de precipitação significativa entre 17 e 34 DAS impossibilitou a aplicação de nitrogênio em cobertura.

$\mathrm{Na}$ avaliação de fitotoxicidade aos 7 DAA (Tabela 2), os tratamentos que continham o herbicida 2,4-D ocasionaram maior injúria ao milheto, em especial ao tratamento com $150 \%$ da dose, $4,5 \%$ e $7,5 \%$, respectivamente, onde o principal sintoma foi de clorose internerval. Na cultura do sorgo, a aplicação do herbicida 2,4-D apresentou sintomas semelhantes (PETTER et al., 2011). Já os tratamentos contendo o herbicida atrazine apresentaram leves sintomas de fitotoxicidade nas plantas, caracterizando-se apenas por clorose internerval, sintoma característico de herbicidas inibidores do fotossistema II (WELLER, 2003).

Tabela 2. Fitotoxicidade aos 7, 14, 21 e 28 DAA, na região Norte do Rio Grande do Sul. IFRS, Sertão, 2020.

\begin{tabular}{lcccc}
\hline \multicolumn{1}{c}{ Tratamento } & \multicolumn{4}{c}{ Fitotoxicidade (\%) } \\
\cline { 2 - 5 } & $7 \mathrm{DAA}$ & $14 \mathrm{DAA}$ & $21 \mathrm{DAA}$ & $28 \mathrm{DAA}$ \\
\hline Testemunha sem capina & $0 \mathrm{~d}^{\mathrm{l}}$ & $0 \mathrm{c}$ & $0 \mathrm{~d}$ & $0 \mathrm{~d}$ \\
Testemunha capinada & $0 \mathrm{~d}$ & $0 \mathrm{c}$ & $0 \mathrm{~d}$ & $0 \mathrm{~d}$ \\
Atrazine em pré-emergência & $0 \mathrm{~d}$ & $0 \mathrm{c}$ & $0 \mathrm{~d}$ & $0 \mathrm{~d}$ \\
Atrazine em pós-emergência & $0 \mathrm{~d}$ & $0 \mathrm{c}$ & $0 \mathrm{~d}$ & $0 \mathrm{~d}$ \\
Atrazine em V4 & $2 \mathrm{c}$ & $3 \mathrm{~b}$ & $3,75 \mathrm{c}$ & $4 \mathrm{c}$ \\
2,4-D em V4 & $4,5 \mathrm{~b}$ & $6,75 \mathrm{a}$ & $8,75 \mathrm{~b}$ & $10,25 \mathrm{~b}$ \\
2,4-D 150\% em V4 & $7,5 \mathrm{a}$ & $9,25 \mathrm{a}$ & $13,5 \mathrm{a}$ & $15,5 \mathrm{a}$ \\
Atrazine em pré-emergência + 2,4-D em V4 & $1,5 \mathrm{~cd}$ & $7 \mathrm{a}$ & $9,25 \mathrm{~b}$ & $10,5 \mathrm{~b}$ \\
Atrazine em pós-emergência + 2,4-D em V4 & $1,75 \mathrm{~cd}$ & $7,25 \mathrm{a}$ & $9,5 \mathrm{~b}$ & $10,75 \mathrm{~b}$ \\
Atrazine + 2,4-D em V4 & $5 \mathrm{~b}$ & $7,25 \mathrm{a}$ & $9,75 \mathrm{~b}$ & $11 \mathrm{~b}$ \\
\hline Média & 2,22 & 4,1 & 5,45 & 6,2 \\
${ }^{2}$ Dms & 1,99 & 2,76 & 2,02 & 1,97 \\
${ }^{3} \mathrm{CV}(\%)$ & 36,77 & 27,61 & 15,19 & 13,06 \\
\hline
\end{tabular}

${ }^{1}$ Médias seguidas pela mesma letra minúscula na vertical não diferem significativamente pelo teste de Tukey a $5 \%$ de probabilidade. ${ }^{2}$ Dms: diferença mínima significativa. ${ }^{3} \mathrm{CV}$ : Coeficiente de Variação. 
$\mathrm{Na}$ avaliação de fitotoxicidade realizada 14,21 e 28 DAA (Tabela 2) os sintomas dos herbicidas se acentuaram, sendo as maiores injúrias causadas pelo herbicida 2,4-D, em especial quando aplicado em $150 \%$ da dose. Na avaliação realizada aos 28 DAA o herbicida $2,4-\mathrm{D}$ a $100 \%$ e $150 \%$ da dose causaram $10,25 \%$ e $15,5 \%$ de fitotoxicidade, respectivamente. A aplicação isolada de atrazine em V4 causou fitotoxicidade de 4\%, o herbicida 2,4-D em 100\% da dose apresentou fitotoxicidade similar quando aplicado isoladamente ou em combinação com o herbicida atrazine. Dan et al. (2011) também relata a baixa fitotoxicidade do herbicida atrazine à cultura do milheto.

$\mathrm{O}$ herbicida 2,4-D apresenta seletividade à maioria das gramíneas, mesmo aplicado em diferentes estádios fenológicos e doses, porém isto não pode ser considerado como regra, pois a variabilidade genética, mesmo dentro de uma própria espécie interfere na sua tolerância ao herbicida em questão (PETERSON et al., 2016). Os principais mecanismos de tolerância ao herbicida 2,4-D são a baixa translocação no xilema e floema, baixa absorção (pilosidade, cutícula espessa, etc.) e detoxificação (PETERSON et al., 2016).

\section{Conclusão}

O herbicida 2,4-D é pouco seletivo ao milheto, causa grande redução na altura de plantas e no acúmulo de matéria seca quando aplicado no estádio V4. O herbicida atrazine apresentou efeito pré-emergente sobre o milheto, aplicado antes e logo após à semeadura, reduzindo o estande de plantas. A aplicação de atrazine no estádio V4 ocasiona pouca fitotoxicidade à cultura, apresentando-se como uma alternativa no controle de plantas daninhas.

\section{Referências}

Berglund, D. R. Proso Millet In North Dakota. Fargo: North Dakota State University, 2007. 7 p. Disponível em: $<$ https://library.ndsu.edu/ir/bitstream/handle/10365/9153/ A805_20 07.pdf?sequence=1>. Acesso em: 12 mar. 2020.

Carson, A. G. Improvement Weed Management In The Draft Animal-Based Production Of Early Pearl Millet In Gambia. Tropical Pest Management, v. 33, n. 02, p. 359363, 1987. Disponível em: <https:/www.tandfonline.com/ doi/abs/10.1080/09670878709371183>. Acesso em: 15 mar. 2020 .

Costa, C. H. M.; Crusciol, C. A. C.; Soratto, R. P.; Ferrari Neto, J. Persistência e liberação de elementos da fitomassa do consórcio crotalária com milheto sob fragmentação. Revista Ciência Agronômica, Fortaleza, v. 45, n. 1, p. 197208, 2014.

Dan, H. A. et al. Tolerância do cultivar de milheto ADR-300 ao herbicida atrazine. Revista Ciência Agronômica, v. 42, n. 01, 2011. Disponível em: <https://www.scielo.br/ scielo.php?p id=S1806-66902011000100024\&script=sci arttext\& tl ng=pt>. Acesso em 02 jul. 2020.

Dantas, C. C. O.; Negrão, F. M. Características agronômicas do Milheto (Pennisetum glaucum). PUBVET, Londrina, v. 4, n. 37, ed. 142, p. 3-4, 2010.

EMBRAPA Trigo Meteorologia: Informações Meteorológicas Diárias. Disponível em: <http:// www.cnpt.embrapa.br/pesquisa/agromet/app/principal/>. Acesso em: 13 mar. 2020.

Fornarolli, D. A.; Rodrigues, B. N.; Lima, J.; Valerio, M. A. Influência da cobertura morta no comportamento do herbicida atrazine. Planta daninha, v. 16, n. 2, p. 97-107, 1998.

Frans, R. W. Measuring plant response. In: Wilkinson, R. E. (Ed.). Research methods in weed science. Puerto Rico: Weed Science Society, 1972. p. 28-41.

Herrada, M. R.; Leandro, W. M.; Ferreira, E. P. B. Leguminosas isoladas e consorciadas com milheto em diferentes sistemas de manejo do solo no feijão orgânico. Terra Latino Americana, v. 35, n.4, p. 293-299, 2017.

Marcante, N. C.; Camacho, M. A.; Paredes, F. P. J. Teores de nutrientes no milheto como cobertura de solo. Bioscience Journal, Uberlândia, v. 27, n. 2, p. 196-204, 2011.

Pacheco, L. P. et al. Tolerância do milheto (Pennisetum americanum) ao 2,4-D. Planta Daninha, v. 25, n. 1, p. 173179, 2007.

Pereira Filho, I. A. et al. Manejo da cultura do milheto. Embrapa Milho e Sorgo, Sete Lagoas, 2003. 17 p. (Embrapa Milho e Sorgo. Circular Técnica, 29). Disponível em: $\quad<$ https:// www.infoteca.cnptia.embrapa.br/infoteca/ bitstream/doc/487545/1/Circ29.pdf $>$. Acesso em: 10 mar. 2020 .

Petter, F. A.; Pacheco, L. P.; Alcântara Neto, F.; Zuffo, A. M.; Procopio, S. O.; Almeida, F. A. Desempenho agronômico do sorgo em função de doses e épocas de aplicação do herbicida 2,4-D. Planta Daninha, Viçosa, v. 29, n. esp., p. 1091-1098, 2011.

Peterson, M. A.; Mcmaster, S. A.; Riechers, D. E.; Skelton, J.; Stahlman, P. W. 2,4-D past, present, and future: a review. Weed Science, Cambridge, v. 30, n. 2, p. 303-345, 2016.

Rodrigues, S. M. Seletividade de Herbicidas Aplicados em Pré-Emergência na Cultura do Sorgo Biomassa. Instituto Federal de Educação, Ciência e Tecnologia Goiano Campus Rio Verde, Rio Verde, 2019. 37 p. Dissertação (Mestrado em Bioenergia e Grãos) - Instituto Federal de Educação, Ciência e Tecnologia Goiano - Campus Rio Verde, 2019. Disponível em: $<$ https:// repositorio.ifgoiano.edu.br/bitstream/prefix/760/3/Disserta $\%$ c3\%a7\%c3\%a3o_S andro\%20Miranda.pdf $>$. Acesso em: 14 
jul. 2020.

Serafim, R. S.; Antonelli, A.; Santos, M. A. T. Determinação da matéria seca e proteína bruta pelo método convencional e microondas. FAZU em Revista, n. 11, 2016.

Weller, S. Herbicide Action Course. West Lafayette:

Purdue University, 2003. P. 267-291. Disponível em: $<$ https://www.scielo.br/scielo.php?scri pt=sci_nlinks\&ref= 000127\&pid $=$ s1806-6690201100010002400027\&lng=em $>$. Acesso em: 13 jul. 2020. 